\section{P31 EVALUATION OF THE RESULTS OF HIP ULTRASOUND SCREENING FOR DEVELOPMENTAL DYSPLASIA OF THE HIP AMONG INFANTS IN A TERTIARY SETTING}

${ }^{1}$ Feyza Aydin, ${ }^{1} E m e l$ Gur, ${ }^{2}$ Tugba Erener-Ercan*, 'Ahmet Arvas. 'Istanbul University, Cerrahpasa Medical Faculty, Istanbul, Turkey; ${ }^{2}$ Maltepe University, Medical Faculty, Istanbul, Turkey

\subsection{6/archdischild-2019-epa.386}

Background The aim of our study was to investigate the rate of developmental dysplasia of the hip (DDH), and the association between the risk factors for DDH and the results of the hip ultrasound (US) findings among healthy infants

Methods The study group consisted of healthy infants who admitted to the outpatient Healthy Child Clinics of Istanbul University, Cerrahpasa Medical Faculty for their routine control between December 2014 and May 2015. Files of the patients who were followed up at least 1 year of age were reviewed with regard to risk factors (sex, birth weight and lenght, type of delivery, order of birth, type of presentation, maternal age, history of oligohydramnios, multiple pregnancy, swaddling history, family history of DDH) and hip US findings. All infants had their hip US performed at 4 to 6 weeks of age in the Radiology Department of Istanbul University.

Results A total of 300 infants $(175 / 125:$ male/female) with a birth weight and length of $3137.03 \pm 557.23 \mathrm{gr}$ and 49.69 $\pm 2.68 \mathrm{~cm}$, respectively were enrolled. Fifteen infants had a history of maternal oligohidramnios (5\%), 70 (23.3\%) were born vaginaly, 27 (9\%) were born as twins and $2(0.7 \%)$ had a breech presentation. Family history of DHH was present in 17 infants (5.7\%) and $28(9.3 \%)$ had a swaddling history. US finding of immature hip was detected in $52.9 \%$ of those with a family history of $\mathrm{DHH}$ and $13.6 \%$ of those with a swaddling history. There was a statistically significant association between family history of $\mathrm{DHH}$ and swaddling, and finding of immature hip on US $(p=0.0001)$. In those with findings of immature hip, left hip was affected in 28 (56\%) and right hip was affected in $13(26 \%)$ infants. Involvement of left hip was significantly more frequent in case of an immature hip on US $(p=0,04)$. Only one infant had a finding of DDH (Type 2b) on US.

Conclusions The rate of $\mathrm{DHH}$ in this study was $0.3 \%$. while the rate of immature hip was $16.7 \%$. Positive family history and swaddling were found to have a strong association with immature hip on US with a significantly more frequent involvement of the left hip. We want to emphasize that family history of DHH should be sought during evaluation of an infant with regard to $\mathrm{DHH}$. We realized that swaddling was still a common practice in Turkey for which the parents should be warned about its associated risk for DHH.

\section{P32 SIGNIFICANCE OF MRI IN THE DIAGNOSIS OF PELVIC OSTEOMYELITIS IN CHILDREN - 2 CASE REPORTS}

${ }^{1}$ Ivan Peychl*, ${ }^{2}$ Jan Hajicek, ${ }^{3}$ Jiri Chomiak, ${ }^{4}$ Jan Lami. ${ }^{1}$ Department of Paediatrics, Na Bulovce Hospital, Prague, Czech Republic; '2Department of Radiology, Na Bulovce Hospital, Prague, Czech Republic, ${ }^{3}$ Department of Orthopaedic Surgery, Na Bulovce Hospital, Prague, Czech Republic; ${ }^{4}$ Nuclear Medicine Centre, Prague, Czech Republic

10.1136/archdischild-2019-epa.387

Case 1 In a 7-year-old boy with right inguinal pain, limp, fever and CRP of $288 \mathrm{mg} / \mathrm{l}$, X-rays and ultrasound of both hips showed normal findings. On day 3 of treatment with ibuprofen and intravenous ceftriaxon MRI of pelvis and hips was performed, showing widening and increased signal of the inferior ramus of the right pubis with oedema of the surrounding obturator muscles. The antibiotics were changed to clindamycin. Skeletal scintigraphy was performed on day 7 with the finding of changes in the right pubis. Within 15 days of treatment, laboratory findings normalised. Clinical symptoms disappeared within 3 weeks. The antibiotic treatment was continued for 6 weeks altogether. The last MRI performed after 10 weeks revealed partial resolution of the oedema of the pubis.

Case 2 13-year-old boy was investigated by his community paediatrician for fever and left inguinal pain. X-rays of the pelvis and lower extremities were normal. We admitted the boy 8 days after the start of fever for the suspicion of acute osteomyelitis. Paediatric orthopedist ruled out coxitis, both $\mathrm{X}$ rays and ultrasound examination of the hips were normal. CT scan of the abdomen, pelvis and the left hip showed normal findings on the bones and muscles. We started treatment with bed rest and intravenous ceftriaxon. On day 3, blood culture proved positive for Staphylococcus aureus and the treatment was switched to intravenous clindamycin. MRI of the pelvis and hips performed on day 4 showed changes in the superior ramus of the left pubis and the obturator muscles. Pain in the left hip disappeared within a week of treatment. Laboratory findings showed an increase of CRP level up to $370 \mathrm{mg} / \mathrm{l}$ on day 3. The level normalised within 10 days of treatment. The boy was discharged home after 24 days of inpatient stay. The treatment continued with 3 weeks of oral cefuroxim. Followup MRI performed 39 days after admission showed partial resolution of the inflammatory oedema of the left superior pubis.

Conclusion Our findings correspond to the 2017 ESPID guidelines: MRI has a primary role in the diagnosis of paediatric acute osteomyelitis. MRI changes of bones and muscles are present in the early stage of the disease and they persist for weeks/months at a minimum. Provided the location of the inflammation is clinically apparent, radionuclide study is not a necessary part of the investigation. CT is of little value in the diagnosis of acute osteomyelitis.

\section{P33 BONE MINERAL DENSITY IN CYSTIC FIBROSIS CHILDREN IN SAINT PETERSBURG}

Aleksandr A Pashkevich*, ${ }^{2}$ Aleksandr V Orlov, ${ }^{3}$ Mariya V Kuropatenko, ${ }^{4}$ Viktor N Kovalev, 'Ludmila A Zhelenina. 'St. Petersburg State Pediatric Medical University, Ministry of Healthcare of the Russian Federation, Saint Petersburg, Russian Federation; ${ }^{2}$ North-Western State Medical University named after I.I. Mechnikov, Saint Petersburg, Russian Federation; ${ }^{3}$ FSBSI «Institute of Experimental Medicine», Saint Petersburg, Russian Federation; ${ }^{4}$ City Children's Hospital Saint Olga, Saint Petersburg, Russian Federation

\subsection{6/archdischild-2019-epa.388}

Background Bone mineral disturbances are often complication of the cystic fibrosis (CF) patients with impact linear growth, quality of life and life expectation and may be contraidication for lung transplant. Osteoporosis is a complex multifactorial diseases which started in the childhood.

The aim of our study was to evaluate bone mineral metabolism in CF children in the Saint-Petersburg.

Materials In the present study $57 \mathrm{CF}$ children, aged 5-18 years were included. For assessment of bone health we evaluated: i) number of significant fractures; ii) dual-energy $\mathrm{X}$ - 
ray absorptiometry (Lunar iDXA 16[SP2]) with pediatric database in lumbar spine (L1-L4) and in total body less head (TBLH); iii) bone age with Greulich-Pyle method; iiii) Tanner's puberty stage and anthropometry. In the cases with significant growth delay or acceleration the height- or biologic age standardization for bone mineral density (BMD) applied

Results The BMD Z score -1SD to - 2SD was in 15 (26.3\%) children and $Z$ score $<-2$ SD in 5 (8.8\%). All children with BMD L1-L4 Z score <-2SD had growth delay so after applying of above mentioned correction methods where were no patients with severe bone mineral density (Zscore $<-2$ SD) deficiency. Only 3 children had decreased (near -2SD) BMD in total body less head program. No patients with pathological low-energy fractures were observed.

Conclusion We have not found osteoporotic CF patients. Further investigations required for cystic fibrosis patients with borderline decreased bone mineral density (near or less than $2 \mathrm{SD})$.

\section{P34 THE LONG-TERM RESULTS OF SINGLE-EVENT MULTILEVEL SURGERY IN TREATMENT OF CHILDREN WITH SPASTIC DIPLEGIA}

${ }^{1}$ Mikhail Herasimenka, ${ }^{2}$ Leanid Hlazkin*, ${ }^{2}$ Mikhail Mikhovich, ${ }^{3}$ Raman Klimau, ${ }^{1}$ Aleh Sakalouski. 'Republican Scientific and Practical Centre for Traumatology and Orthopaedics, Minsk, Belarus; ${ }^{2}$ Mogilev Regional Children's Hospital, Mogilev, Belarus; ${ }^{3}$ Brest Regional Children's Hospital, Brest, Belarus

\subsection{6/archdischild-2019-epa.389}

Introduction Cerebral palsy is a group of permanent disorders of the development of movement and posture, causing activity limitation, that are attributed to non-progressive disturbances that occurred in the developing fetal or infant brain. Single-Event Multilevel Surgery - SEMLS has been used by most surgeons for correction limb deformities in the past decades.

The aim Of our study was to evaluate the long-term results of SEMLS, performed in the Mogilev Regional Children's hospital and in the Republican Scientific and Practical Centre for Traumatology and Orthopaedics.

Materials and methods The long-term results of SEMLS in 19 children with spastic diplegia, operated in 2009-2016 were studied. All the patients had spastic deformities of the hips, knees and ankles on the both sides before surgery. All the patients were GMFCS IV. The mean age at time of surgery was 6,7. 138 procedures were performed. All the procedures were made simultaneously on the both sides by two teams of surgeons. Observational Gait Scale (OGS) and Edinburgh Visual Gait Score (EVGS) were used to determine the effectiveness of treatment.

Results The minimal follow-up was 3 years. The mean improvement by OGS was 8,5 , the minimal improvement -3 , maximal - 12. The mean improvement by EVGS was 10,1 , the minimal improvement -6 , maximal -16 . All the parents noticed the improvements in child's mobility after surgery. One patient has severe valgus foot deformity and calcaneal gait after anterior Achilles tendon transfer as part of SEMLS in 2015. Six patients underwent additional surgery for correction of the secondary bone deformities later.

Conclusions Brain disorders in cerebral palsy are permanent and as S.Terver said - 'The child with cerebral palsy becomes the adult with cerebral palsy'. For the child with cerebral palsy the main goals of surgical treatment - improving the child's motor activity and elimination of severe limb deformities.

SEMLS changes the whole pathological stereotype of musculoskeletal balance of extremities. It can improve the child's motor activity, eliminate the severe contractures of joints, provide adequate care for him in a relatively short period of time and save these improvements in the longterm follow-up. Care must be taken in choosing the proper method of surgical correction, especially in treatment of equinus.

\section{P35 IMPROVED SURVIVAL IN RARE RECESSIVE OSTEOGENESIS IMPERFECTA THROUGH MULTIDISCIPLINARY TEAM SUPPORT AND EARLY BISPHOSPHONATE USE}

${ }^{1}$ Gillian O'Donnell*, 'Laura C McCarron, 'Eleanor Burke, ${ }^{1,2}$ Ciara M McDonnell. 'Department of Paediatric Endocrinology, Children's University Hospital, Temple St, Dublin, Ireland; '2Discipline of Paediatrics, University of Dublin, Trinity College, Dublin, Ireland

\subsection{6/archdischild-2019-epa.390}

Osteogenesis imperfecta (OI) or Brittle bone disease is a rare genetic connective tissue disorder with the majority of mutations found in collagen type 1 genes (COL1A1/ COL1A2) or their related SIBLING proteins. The condition is characterized by increased bone fragility, resulting from abnormal collagen formation. Recessive forms of OI are associated with increased severity and lethality due to mutations in LEPRE1, encoding prolyl 3-hydroxylase-1 (P3H1) or in CRTAP, encoding cartilage associated protein. The LEPRE1 gene mutation has been expressed in the Irish Traveller population. We report two children affected with this mutation who have demonstrated a positive response to early bisphosphonate use.

Case 1 A male infant was born in 2013 by elective LSCS at 38 weeks gestation to consanguineous Caucasian parents from the Irish traveller community. Antenatal scans in the third trimester had confirmed multiple in utero fractures. He was commenced on Pamidronate (Bisphosphonate) infusion at four weeks of age. He now sits and transfers independently and is attending school.He has sustained further fractures with trauma.

Case 2 A male infant was born in 2018 by elective LSCS at 39 weeks gestation to consanguineous Caucasian parents from a separate family tribe within the Irish traveller community. Antenatal scans at 22 weeks confirmed limited femur growth and the possibility of skeletal dysplasia were raised at 23 weeks when significant fractures were noted. There was a strong family history of osteogenesis imperfecta with the death of four of his mother's siblings in childhood with a clinical diagnosis of brittle bone disease. She was believed to be unaffected having never sustained a fracture.He was commenced on an initial dose of zoledronic acid on Day 7 of life with a subsequent reduction in analgesia requirement and increase in peripheral limb activity. He was discharged home at 4 weeks of age.

Discussion Molecular genetics confirmed a diagnosis of type VIII OI caused by a pathogenic mutation in the LEPRE1 (P3H1) gene in both cases. Skeletal survey at birth showed thin ribs, multiple wormian bones and healing fractures of the clavicles, ribs, humeri and femori. Both exhibited a 